\title{
Detection of a repeated transit signature in the light curve of the enigma star KIC 8462852: A possible 928-day period
}

\author{
F. Kiefer ${ }^{1}$, A. Lecavelier des Étangs ${ }^{1}$, A. Vidal-Madjar ${ }^{1}$, G. Hébrard $^{1,2}$, V. Bourrier ${ }^{3}$, and P. A. Wilson ${ }^{1,4}$ \\ 1 Institut d'Astrophysique de Paris, UMR 7095 CNRS, Université Pierre \& Marie Curie, 98 bis boulevard Arago, 75014 Paris, France \\ e-mail: flavien.kiefer@iap.fr \\ 2 Observatoire de Haute-Provence, CNRS, Université d'Aix-Marseille, 04870 Saint-Michel-l'Observatoire, France \\ 3 Observatoire de Genève, Chemin des Maillettes 51, Sauverny, 1290 Versoix, Switzerland \\ ${ }^{4}$ Leiden Observatory, Leiden University, Postbus 9513, 2300 RA Leiden, The Netherlands
}

Received 2 June 2017 / Accepted 2 September 2017

\begin{abstract}
As revealed by its peculiar Kepler light curve, the enigmatic star KIC 8462852 undergoes short and deep flux dimmings at a priori unrelated epochs. This star presents nonetheless all other characteristics of a quiet $1 \mathrm{Gyr}$ old F3V star. These dimmings resemble the absorption features expected for the transit of dust cometary tails. The exocomet scenario is therefore most commonly advocated. We reanalysed the Kepler data and extracted a new high-quality light curve to allow for the search of shallow signatures of single or a few exocomets. We discovered that among the 22 flux dimming events that we identified, two events present a striking similarity. These events occurred 928.25 days apart and lasted for 4.4 days with a drop in the star brightness by 1000 ppm. We show that the light curve of these events is well explained by the occultation of the star by a giant ring system or by the transit of a string of half a dozen exocomets with a typical dust production rate of $10^{5}-10^{6} \mathrm{~kg} \mathrm{~s}^{-1}$. Assuming that these two similar events are related to the transit of the same object, we derive a period of 928.25 days. The following transit was expected in March 2017 but bad weather prohibited us from detecting it from ground-based spectroscopy. We predict that the next event will occur between 3-8 October 2019.
\end{abstract}

Key words. stars: individual: KIC 8462852 - techniques: photometric - comets: general - planets and satellites: rings

\section{Introduction}

KIC 8462852 is a peculiar and intriguing source that garnered a lot of attention from the astronomical community in the recent years (Boyajian et al. 2016; Marengo et al. 2015; Lisse et al. 2015; Schaefer 2016; Hippke et al. 2016; Abeysekara et al. 2016; Bodman \& Quillen 2016; Lund et al. 2016; Thompson et al. 2016; Schuetz et al. 2016; Harp et al. 2016; Wright \& Sigurdsson 2016; Montet \& Simon 2016; Makarov \& Goldin 2016; Neslušan \& Budaj 2017; Ballesteros et al. 2018). KIC 8462852 is an F3V star located at about $454 \pm 35 \mathrm{pc}$ away from Earth (Hippke \& Angerhausen 2016). The Kepler spacecraft photometric data revealed an enigmatic light curve for this star with erratic, up to $\sim 20 \%$ deep, stellar flux dimmings (Boyajian et al. 2016). Because it is otherwise considered a standard F star, stellar instabilities could be excluded as an explanation for its strange behaviour. More recently, it was found that the flux of KIC 8462852 dropped by $\sim 2.5 \%$ over 200 days during the Kepler mission (Montet \& Simon 2016), while a thorough analysis of photographic plates taken over the last century seemed to show a continuous decrease of the flux by about $0.3 \% \mathrm{yr}^{-1}$ (Schaefer 2016). The study of Schaefer (2016) was however later on refuted by Hippke et al. (2016, 2017) and Lund et al. (2016).

The most popular scenario that has been advocated to explain the frequent but aperiodic dips is that of many uncorrelated circumstellar objects transiting at different epochs; i.e. either comets (Boyajian et al. 2016; Neslušan \& Budaj 2017) or planetesimal fragments (Bodman \& Quillen 2016). This is reminiscent of the case of $\beta$ Pictoris; many variable narrow absorptions were observed on the high resolution spectra of $\beta$ Pictoris in Ca II doublet, which are best explained by extrasolar comets, also known as exocomets (Ferlet et al. 1987; Beust et al. 1990; Kiefer et al. 2014a). However, in contrast to $\beta$ Pictoris, KIC 8462852 is not young ( $1 \mathrm{Gyr}$ ) and any circumstellar gas or dust remain unobserved at infrared wavelengths.

About 20 years ago, Lecavelier Des Etangs et al. (1999) published innovative simulations of photometric signatures produced by the transit of the dusty tail of exocomets. The shape of the theoretical absorption signatures obtained has unique characteristics: a peaky core for the transit of the head of the coma and a long trailing slope. Nonetheless, the only direct evidence for comets around stars other than the Sun came from high-resolution spectroscopy observations of the atomic gas counterparts of cometary tails in the disks of, e.g. $\beta$ Pic (Ferlet et al. 1987), HD172555 (Kiefer et al. 2014b), HR10 (Lagrange-Henri et al. 1990) or 49 Ceti (Montgomery \& Welsh 2012; Miles et al. 2016). Before KIC 8462852, photometry never revealed any direct observations of exocomets around any star.

The level of precision needed to detect the transit of a single $\beta$ Pic-like exocomet is about several 100 ppm (Lecavelier Des Etangs et al. 1999). Detecting such an object is a difficult task since a single solar-like exocomet cannot be expected to transit several times during the lifetime of Kepler; in the solar system, comets have periods that are typically larger than three years. Nevertheless, the opportunity of detecting repeated transits should not be completely excluded. We selected KIC 8462852 for thorough analysis of its Kepler light curve with the goal of finding single object $100 \mathrm{ppm}$-deep 
transit signatures. We report in the present paper the detection of a $1000 \mathrm{ppm}$ deep signature repeating twice at a 928-day interval in the KIC 8462852 light curve. We successfully modelled this signature by a string of exocomets crossing the line of sight one after another at $0.3 \mathrm{AU}$ from the central star.

Alternatively, we found that at least one other scenario could provide a good fit of the light curve: the transit of a wide ring system surrounding a planet orbiting at $2.1 \mathrm{au}$ from the star. The Hill sphere could indeed become much wider than the star itself at distances larger than 1 au and could contain transiting materials such as rings (Kenworthy \& Mamajek 2015; Lecavelier Des Etangs et al. 2017; Aizawa et al. 2017). Moreover, while plausible and straightforward, such a scenario was recently proposed by Ballesteros et al. (2018) to explain the smooth and solitary D800 dip of Boyajian et al. (2016) with a transiting ring planet on a 12-year orbit around the star. Thus, instead of being a comet host, KIC 8462852 might just be a planetary system with at least two ring planets.

Kepler data reduction is presented in Sect. 2. The two identical $1000 \mathrm{ppm}$ deep events are presented in Sect. 3. In Sect. 4 we show that these events are real and not instrumental or due to background objects. The models of these events are presented in Sect. 5. Finally, in Sect. 6, we present an attempt to observe the event in March 2017, which failed due to bad weather, and a prediction of its future realizations in October 2019 and later.

\section{Kepler photometric data reduction}

The Kepler spatial observatory (Borucki et al. 2010) followed KIC 8462852 in long cadence mode (30-min sampling) during roughly four years from 2 May 2009 to 11 May 2013, separated into 17 quarters of continuous integration. The Kepler pipeline produced raw (simple aperture photometry, hereafter SAP) and reduced (pre-search data conditioning; PDC-SAP) light curves of the full four-year time range (Smith et al. 2012). The SAP data essentially consist in calibrated flux but uncorrected of cosmic ray absorption, systematic behaviours, jumps etc. The PDCSAP light curves are systematically corrected by the reduction pipeline for every trend of non-astrophysical origin. While the PDCSAP data are certainly good enough to detect short $0.1-1 \%$ deep transits, these data do not reach the level of precision needed to detect $0.1 \%$ deep, possibly day-long, absorption signatures that could be typically produced by transiting exocomets (Lecavelier Des Etangs et al. 1999; Kiefer et al. 2014a). We thus wrote our own MATLAB routine to reduce the SAP light curves carefully; the principles of this routine are explained below.

The Kepler pipeline determines for each quarter, in each CCD channel, an ensemble of 16 cotrending basis vectors (CBV) calculated from the light curves of the brightest stars in the channel using principal component analysis (PCA; Kinemuchi et al. 2012; Smith et al. 2012). These CBVs represent the main systematic behaviours common in the light curves collected on the same channel during the same quarter. For each quarter, we fitted a linear combination of the first $13 \mathrm{CBVs}$ to the SAP light curve of KIC 8462852. Following the recommended process (Kinemuchi et al. 2012), we iteratively increased the number of fitted CBVs. We stopped when the resulting light curve baseline was the flattest in the quiet periods, and when adding more CBVs led to no significant improvement. In order to avoid fitting out the physical dips, we iteratively excluded from the fit any data points below the continuum minus $2 \sigma$, with $\sigma$ defined as

$\sigma=1.48 \times \operatorname{MAD}($ data continuum $)$.
The median absolute deviation (MAD) times 1.48 is an estimation of the standard deviation that is not biased by outliers. Jumps and spikes (cosmics, etc.) were carefully filtered out before applying the fit. We accomplished this by studying the first derivative of the light curve and identifying spikes and jumps signatures. These appear as single or P-cygni shaped 3-5 cadence-long peaks with an amplitude at least four times larger than the local typical cadence-to-cadence variations. Between zero and 10 measurements were found in such discontinuities per quarter. If we encountered a spike, we first removed the bad cadences and then linearly interpolated the light curve through the resultant gap. If we encountered a jump, we removed the bad cadences and separated the light curve into two pieces around the gap; in this case, the CBVs were fitted out to each piece separately.

More generally, anytime there is missing data (typically more than 25 adjacent cadences) we separated the light curve into two pieces around the gap and fitted out CBVs independently for these two pieces. Most of these large discontinuities are due to a monthly Earth downlink and are usually followed by thermal relaxation (Kinemuchi et al. 2012). Even though most of the time the CBVs captured such variations, a simple fitting that ignored the cadences within the gap was not accurate enough. Separating the light curve around these discontinuities led to better results.

Several examples of the detrending results are shown in Fig. 1. We compare these results to the pipeline automatic PDC reduction, which in general presents quarter-long low amplitude variations along the curve and especially around strong dips. With our reduction the continuum is flat, allowing the shallower dips to emerge more evidently than in the PDCSAP data. This shows the positive effect of excluding the measurements of the dips when fitting out the systematics. The full detrended light curve is plotted in Fig. 2.

\section{Two identical photometric shallow events}

Using the light curve obtained in the previous section, we can identify the photometric events that happened during the four years of observation. The observed photometric variations show two different patterns: periodic-like variations and short-time decreases of star brightness. With a period close to 1 day, the periodic modulation corresponds to the 0.88 -day signal due to stellar rotation that was already mentioned by Boyajian et al. (2016). Beyond these variations, the stars show significant short-time and sporadic variations, all of which are dips of the star brightness below the mean brightness observed during the quiet period.

We screen the entire light curve and identified a total of 22 significant dips. Apart from the strong dips already listed by Boyajian et al. (2016), we found several shallower dips, some of which are also identified by Makarov \& Goldin (2016). Table 1 summarizes these detections.

Among the detected features, two events show a remarkable similarity in shape, duration, and depth: the events \#2 and \#13 in Table 1. Hereafter, we label these events as "event A" and "event B". The light curves of these two events are plotted in Fig. 3. In this figure, we superimposed the raw SAPs, fitted CBVs, and corrected SAPs, showing that the two photometric dips are real and not produced by the data analysis procedure.

As indicated in Table 1, events A and B were already noticed by Makarov \& Goldin (2016) but they were suspected to be due to either the centroid modulation of the point spread function (PSF) during event A, or instrumental jitter (event B). In Sect. 4, 


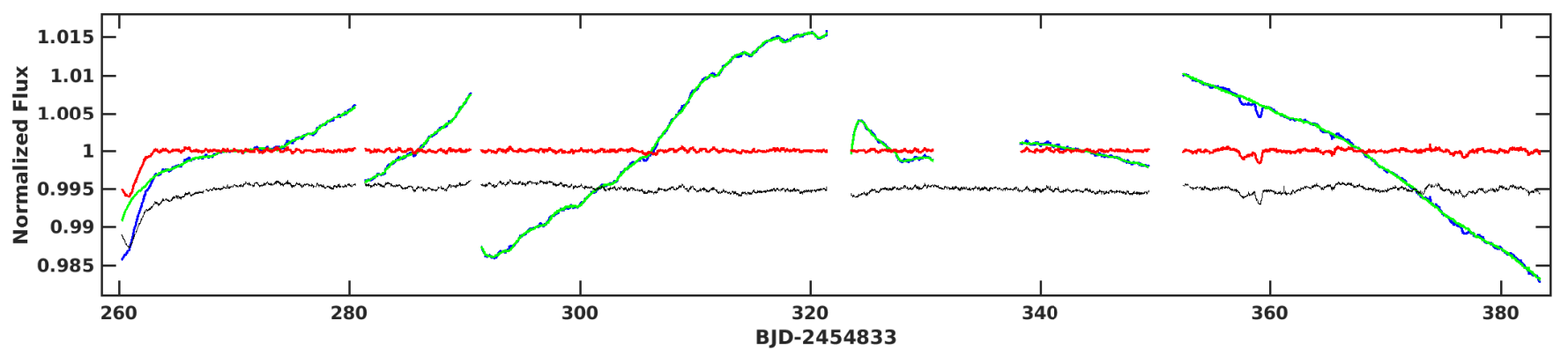

Fig. 1. Example of CBVs fit on light curves with small or no dips. The raw SAP light curve is indicated in blue, the fitted CBVs are indicated in green, and the final detrended SAP light curve is indicated in red. For comparison, we superimposed in black the PDCSAP data with an offset of -0.005 for visual convenience. The green and blue curves overlap most of the time, but it can be seen on some shallow dips (e.g. at BJD-2 $454833=360$ ) that the fitted CBVs, shown in green, stay at the baseline level during these events.

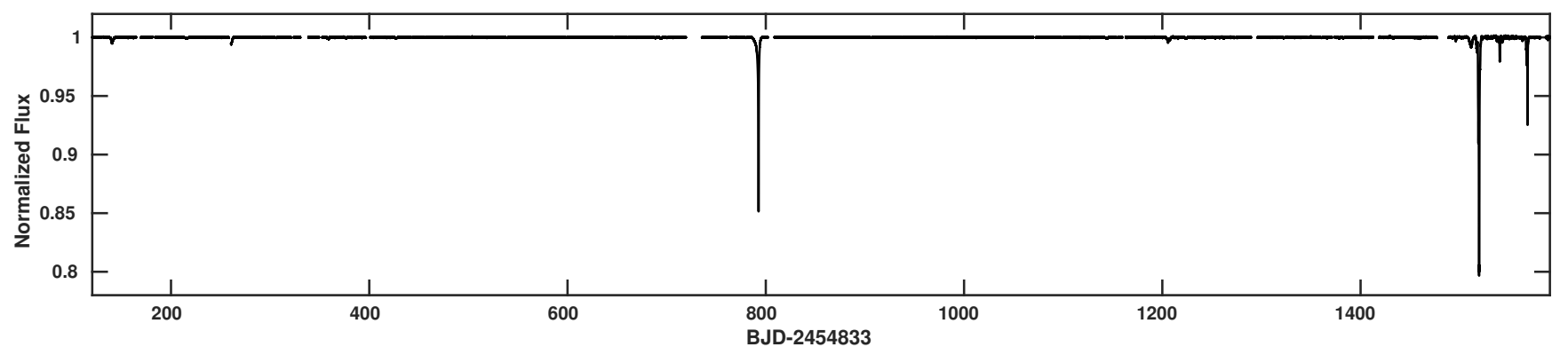

Fig. 2. Full KIC 8462852 detrended light curve.
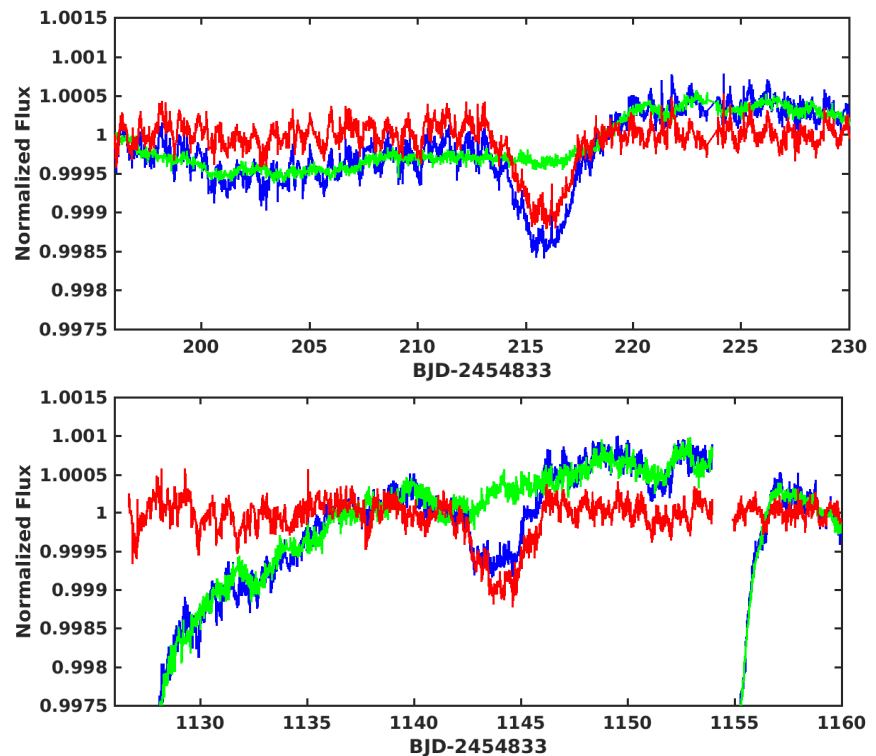

Fig. 3. Light curves at the time of the photometric events A (top panel) and B (bottom panel). The curves show the detrended (red) and undetrended (blue) data sets. The fitted CBVs continuum is plotted with a green line.

we show that these events are of astrophysical origin and are not related to instrumental systematics.

Fitting events A and B together, we derived a time separation between them of $\Delta t=928.25 \pm 0.25$ days. The error bars on the flux were scaled to obtain a reduced $\chi^{2}$ of 1 . Shifting the second event light curve by $-\Delta t$, i.e. on top of the first event light curve, we obtained a strikingly almost perfect superimposition of the two events as shown in Fig. 4.

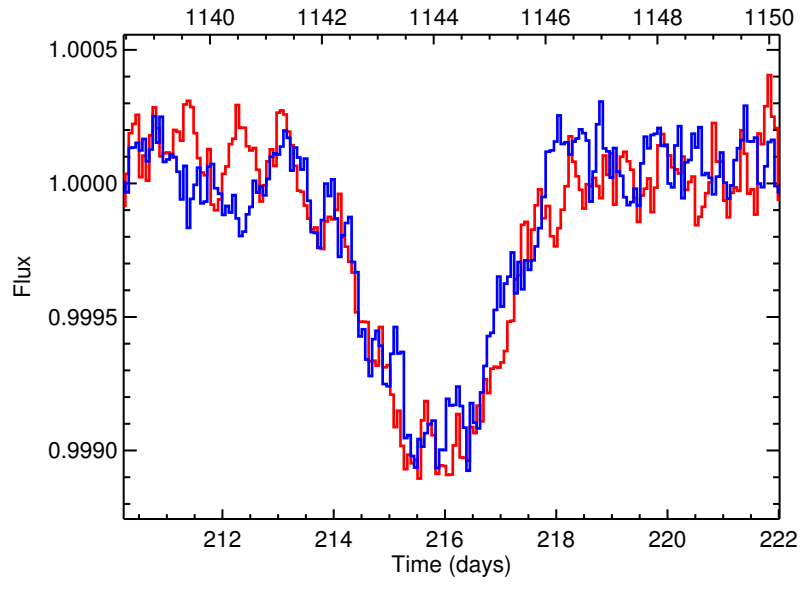

Fig. 4. Two events superimposed with 3-pixel binning. The bottom $x$-axis shows the time at the first photometric event A (red line). The top $x$-axis shows the time at the second photometric event B (blue line) with a shift of 928.25 days relative to the bottom axis.

To characterize the similarity of these two photometric events and compare this pair with the 22 detected events, we plotted the depth and duration of each of the events (Fig. 5). The depths are measured between the continuum level fixed to 1 and the bottom of the light curve defined as the fifth lowest pixel. The durations are measured by calculating the second moments of the variations, which are then multiplied by $2 \sqrt{2 \ln 2}$ to roughly correspond to the full width at half maximum. While other photometric events show a wide diversity in duration and depth, events A and B are remarkably identical. To emphasize this result, we superimposed the light curves of the photometric events \#6 and \#9, which are, after the events A and B, the closest 
Table 1. List of detected photometric dips in the KIC 8462852 light curve.

\begin{tabular}{|c|c|c|c|c|c|}
\hline Event & $\begin{array}{c}\text { Epoch } \\
\text { (JD-2 454 833) }\end{array}$ & $\begin{array}{c}\text { Depth } \\
\left(\log _{10} \Delta F / F\right)\end{array}$ & $\begin{array}{l}\text { Width } \\
\text { (day) }\end{array}$ & Comment & Previous publications ${ }^{\dagger}$ \\
\hline 1 & 140.7 & -2.30 & 1.70 & cometary tail shape (triangular) & B2016, MG2016 \\
\hline 2/A & 215.8 & -2.96 & 1.98 & similar to event 13 & MG2016: modulation of PSF centroid \\
\hline 3 & 261.0 & -2.23 & 1.19 & cut by a gap & B2016, MG2016 \\
\hline 4 & 357.9 & -3.03 & 0.70 & cometary tail shape (triangular) & \\
\hline 5 & 359.0 & -2.82 & 0.41 & superimposed on event 4 & B2016 \\
\hline 6 & 376.6 & -3.08 & 1.25 & noisy surrounding & MG2016 \\
\hline 7 & 427.1 & -3.10 & 1.78 & partly fitted by CBVs & B2016, MG2016 \\
\hline 8 & 688.6 & -3.18 & 0.68 & & \\
\hline 9 & 694.3 & -3.03 & 1.18 & & \\
\hline 10 & 700.6 & -3.32 & 1.72 & series of 4 small dips & \\
\hline 11 & 706.7 & -3.37 & 0.94 & & \\
\hline 12 & 792.6 & -0.90 & 0.77 & very deep event & B2016, MG2016 \\
\hline 13/B & 1144.1 & -2.97 & 1.98 & similar to event 2 & MG2016: instrumental jitter \\
\hline 14 & 1206.2 & -2.38 & 2.54 & narrow event upon a wide event & B2016, MG2016 \\
\hline 15 & 1224.0 & -3.02 & 1.63 & shallow event & \\
\hline 16 & 1496.0 & -2.60 & 0.55 & cometary tail shape (triangular) & B2016 \\
\hline 17 & 1511.4 & -2.10 & 2.24 & preceding a much deeper event & \\
\hline 18 & 1519.4 & -0.71 & 1.00 & very deep event & B2016, MG2016 \\
\hline 19 & 1540.4 & -1.75 & 0.43 & deep event & B2016, MG2016 \\
\hline 20 & 1542.9 & -2.43 & 0.73 & triangular & \\
\hline 21 & 1563.7 & -2.60 & 0.89 & cometary tail shape (triangular) & \\
\hline 22 & 1568.2 & -1.21 & 1.03 & deep event & B2016, MG2016 \\
\hline
\end{tabular}

Notes. Events 2 and 13 are renamed A and B, respectively, in the rest of the paper. ${ }^{(\dagger)}$ MG2016 = Makarov \& Goldin (2016), Table 1; B2016 = Boyajian et al. (2016), Table 1.

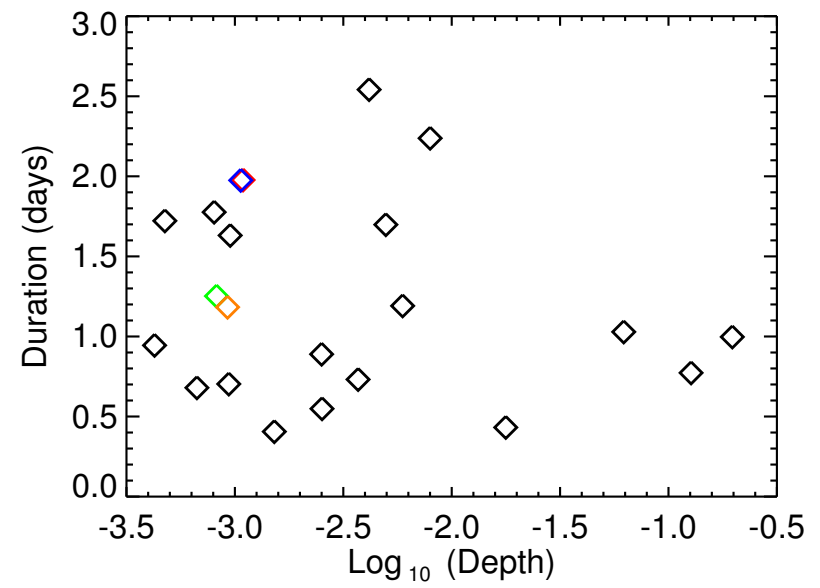

Fig. 5. Depth and duration of the 22 events catalogued in Table 1. The events $\mathrm{A}$ and $\mathrm{B}$ are shown by red and blue symbols, respectively. The events \#6 and \#9 are shown by green and orange symbols, respectively.

in the depth-duration diagram (Fig. 6). It is clear that, unlike events $\mathrm{A}$ and $\mathrm{B}$, these photometric events do not show similar light curve shapes.

The light curve shapes of events A and B can be obtained by fitting a simple four-vertices polygon to each curve. We measured the quantities such as ingress, egress, and centroid timings, slopes of the left and right wings, and transit depth (Table 2). These simple fits quantitatively confirm that the two events are strikingly similar. The average duration of the two events from ingress to egress is measured to be $4.44 \pm 0.11$ days. The bottom of the light curves are flat with a duration of about 1 day. The two slopes on each side of the flat bottom are straight with comparable duration between 1.5 and 2 days. The right wings are steeper

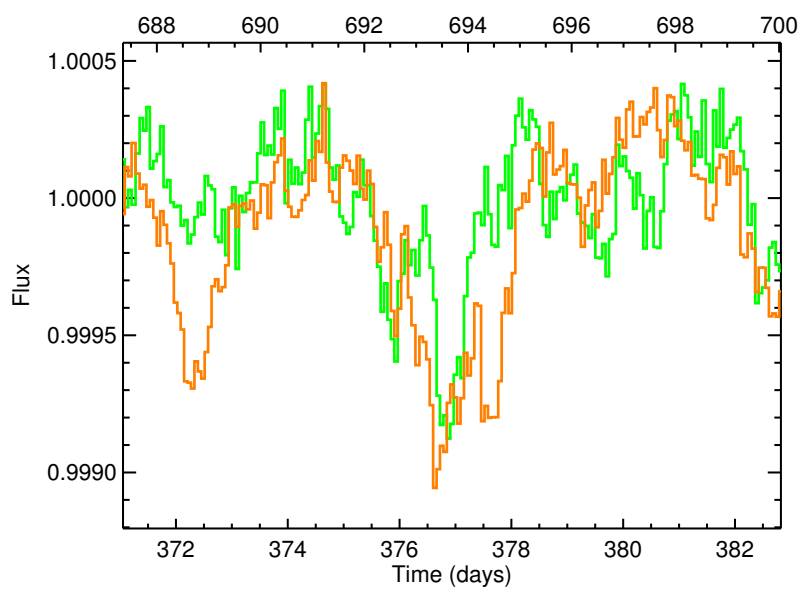

Fig. 6. Events \#6 and \#9 superimposed with 3-pixel binning. The bottom $x$-axis shows the time at the first photometric event \#6 (green line). The top $x$-axis shows the time at the second photometric event \#9 (orange line) with a shift of 316.3 days relative to the bottom axis.

than the left wings with respective slopes of about $700 \mathrm{ppm} /$ day and $-500 \mathrm{ppm} /$ day. The transit depths are similar in both events at about $1010 \pm 40 \mathrm{ppm}$.

If real, these similar events could be the repeated observation of the same periodic phenomenon. After checking for potential reduction artefacts and other systematics in the next section, we discuss interpretations of this repeating event in Sect. 5.

\section{Possible bias}

Makarov \& Goldin (2016) argued that some of the small amplitude features could be of instrumental or background origin. 
Table 2. Four-vertices polygon parameters of the fit to the events A and B light curves.

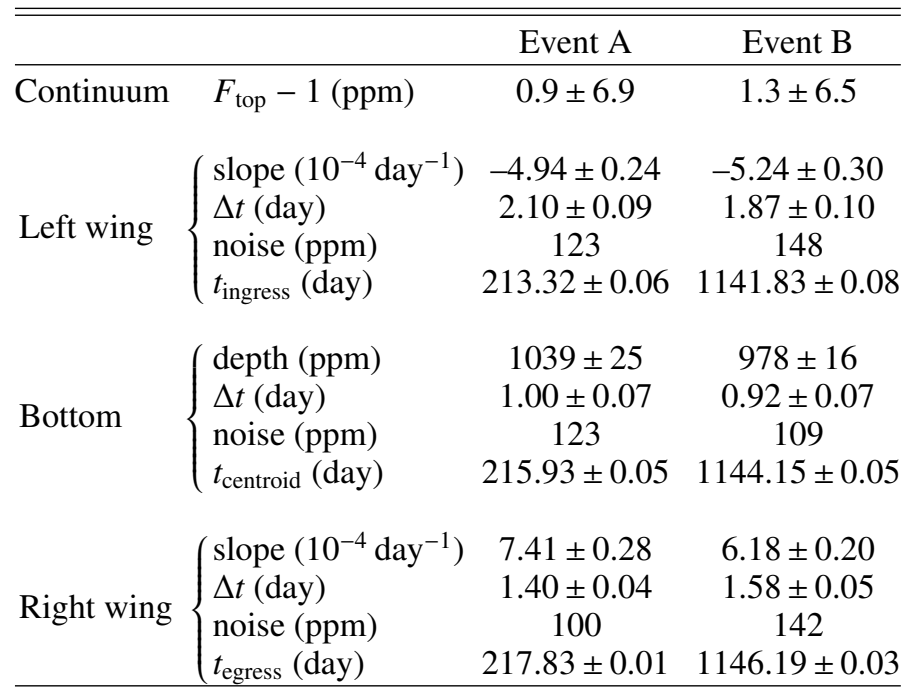

Notes. We used a 4-degree polynomial to fit out the baseline and assumed that the bottom of the light curve is flat. Beginning-of-ingress, centroid, and end-of-egress timings are given in days past Kepler initial epoch at MJD 2454833.

We thus carefully inspected the pixel tables collected by Kepler around the PSF of the star and used to produce the raw SAP light curve about the epochs of the two events to exclude any instrumental origin for these events or the possibility of contamination from close background stars.

\subsection{Background stars}

The closest known stars in the field are Gaia 208190073864563 1744 (at 5.4" with $m_{\mathrm{G}}=18.1$ ), which is referred to as Gaia-208 in the following, and the infrared sources 2MASS $\mathrm{J} 20061551+4427330$ (at 8.9" with $m_{\mathrm{J}}=16.1, m_{\mathrm{G}}=18.9$ ) and 2MASS J20061594+4427365 (at $11.83^{\prime \prime}$ with $m_{\mathrm{J}}=16.4$, $\left.m_{\mathrm{G}}=18.7\right)$. Their high visual magnitude measured by Gaia (van Leeuwen et al. 2017) implies a $\Delta V>6.4$ with KIC $8462852\left(m_{\mathrm{G}}=11.7\right)$, i.e. a flux ratio $<0.3 \%$.

As can be seen in Fig. 7, the pixels corresponding to the theoretical location of the two IR sources in the CCD channel are not within the aperture used to calculate the raw light curve. We see that the flux of KIC 8462852 is smeared on an area about $10 \times 10 \operatorname{arcsec}^{2}$ wide. Since the PSF of the two red stars is likely of similar extension, a bit less than half the flux of 2MASS J20061551+4427330 enters the PSF, while there is almost none for 2MASS J20061594+4427365. Consequently, any flux variation of these stars of order $100 \%$ contaminates the flux to a level lower than $0.1 \%$.

However, since the PSF of Gaia-208 almost fully overlaps with the PSF of KIC 8462852, the photometric variations of this polluting star might induce variations in the light curve, but in any case these variations would not be higher than $0.3 \%$.

At this stage, while we are able to exclude contamination from the two IR background stars, contamination from Gaia-208 cannot be ruled out, although likely to be negligible. In Sect. 4.3, we show that no significant and correlated PSF motion of KIC 8462852 is observed during the two events; this advocates for rejecting contamination from any background stars.

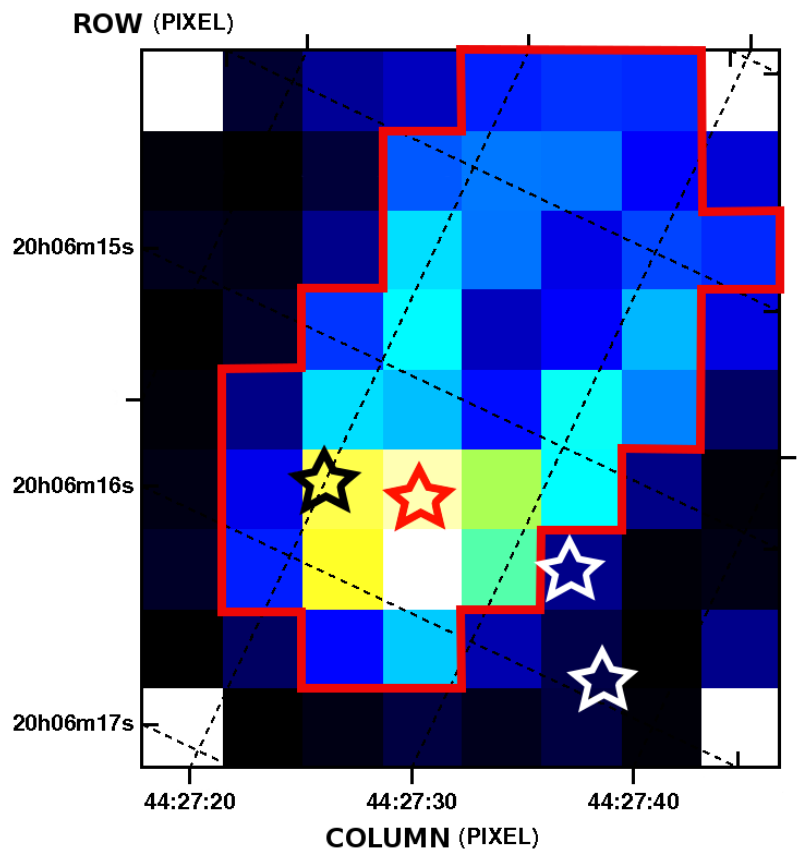

Fig. 7. Pixel table of flux around KIC 8462852, collected during quarter \# 2 on CCD channel 32 . The colours are logarithmically scaled with the flux. The aperture is shown in solid red line. A right ascension and declination grid is superimposed with dotted black lines. The theoretical position of KIC 8462852 on the CCD is depicted as a red star; the position of the two faint IR sources 2MASS J20061551+4427330 \& 2MASS J20061594+4427365 are indicated by white stars; and Gaia 2081900738645631744 appears as a black star.

\subsection{Light curve of closest neighbour KIC 8462934}

KIC 8462934 is the closest bright star (about 89" with $V \sim 11.5$ ) to $\operatorname{KIC} 8462852(V=12)$ with a recorded light curve in the Kepler database. Applying our previously introduced detrending method, we recovered a detrended light curve using the first 13 CBVs of each quarter/channel, as was applied to light curve of KIC 8462852 (see Sect. 2). We found no peculiar behaviour, neither strong nor shallow absorptions similar to what we observed in KIC 8462852. No features were detected in the light curve beyond a few $10^{-4}$ in normalized flux. This indicates that the CBVs derived by the Kepler pipeline did not miss any small local variations in, e.g. pixel sensitivity, in the CCD channel. Therefore, events $\mathrm{A}$ and $\mathrm{B}$ are indeed features from the local pixels in the photometric aperture shown in Fig. 7.

\subsection{Point spread function motion of KIC 8462852 on CCD pixels}

Makarov \& Goldin (2016) studied the correlation of the PSF centroid motion and the flux dimming in KIC 8462852. They show that a few features could be artifacts of occultation or instrumental jitter of background objects.

Repeating a similar analysis on the pixel images collected by Kepler at each cadence, such as presented in Fig. 7, we derived the centroid motion of the PSF of KIC 8462852 before, during, and after the epoch of each identified event. Apart from an expected slow shift $\left(0.004\right.$ pixel day $\left.{ }^{-1}\right)$ of the location of the star on the CCD channel during the quarter, we observed that the centroid oscillates with a period of about three days and an amplitude of at most a few 0.001 pixel (Fig. 8). This oscillation is likely related to a vibration mode of the instrument. Consistently, 

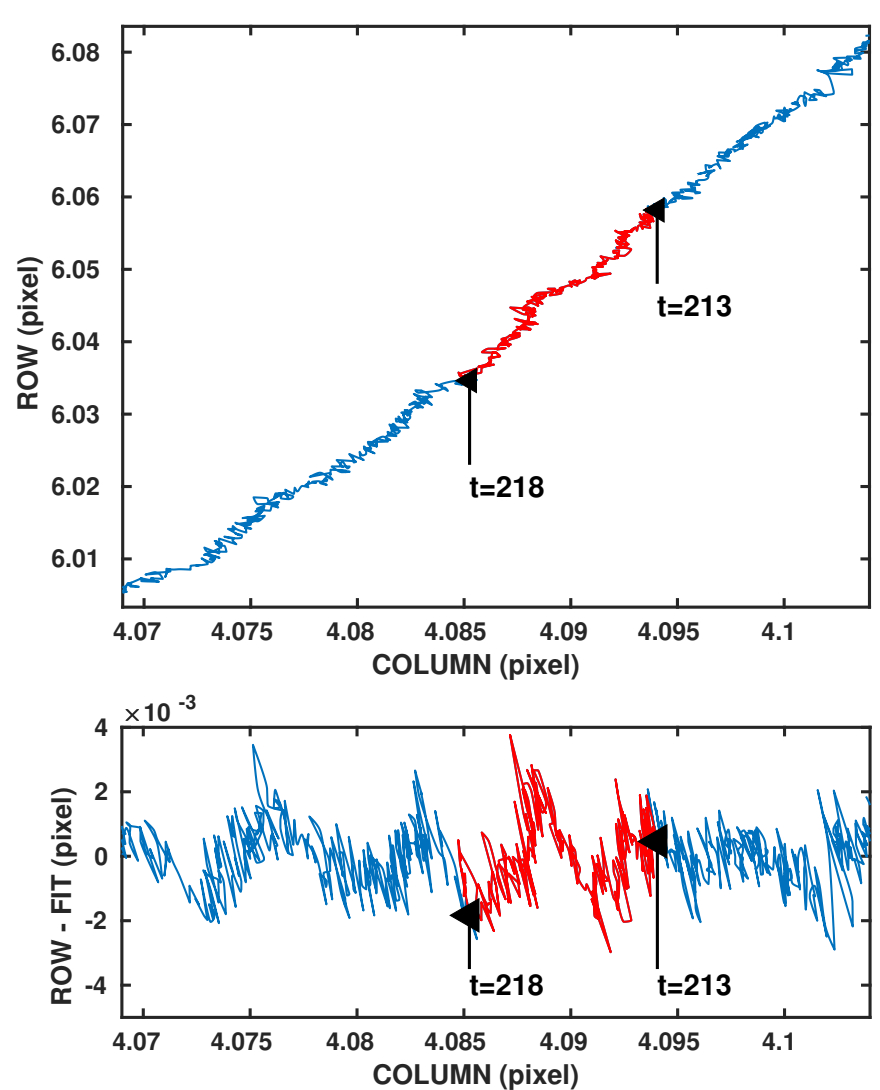

Fig. 8. Top panel: position of the PSF centroid around event A, from epochs 208 to 225 (in blue), and highlighted in red, from ingress (213) to egress (220). Time arrow goes from top right to bottom left. Bottom panel: PSF centroid motion around the 4th degree polynomial fit of the main trend in the top panel. The modulation period is about 3 days.

it was found that a few pixels show shallow flux modulations of a few $0.1 \%$ in correlation or anti-correlation with the PSF centroid oscillations.

Impressively, these instrumental modulations exactly cancel out. We found no counterpart for these modulations in the raw light curve, demonstrating the excellent quality of the flat-field determination made by Kepler on the aperture. Since the amplitude of the three-day modulation is similar to the amplitude of the two events discussed in this paper, instrumental PSF variations cannot be at their origin. Indeed, in such a case, we would have observed $0.1 \%$ deep three-day modulation rather than only single dips.

We have seen in Sect. 4.1 that the closest background star, Gaia-208, is located at 5.4" from KIC 8462852. This is a bit more than 1 pixel apart, on the aperture (Fig. 7). The luminosity variation due to the occultation of a third of Gaia-208 stellar disk would be close to $0.1 \%$, leading to a PSF centroid variation of about $10^{-3}$ pixels. We can exclude from Fig. 8 a PSF centroid variation of this amplitude during event A between day 213.3 and day 217.8 .

Repeating this analysis for event B led to the same conclusion. We thus exclude for both events any significant PSF motion correlated with the light curve, eliminating background star pollution and instrumental variations as possible origins.

\subsection{Local pixel variations}

As a final check, we verified the collective variations of the local flux in each pixel around the PSF of KIC 8462852 at different times between the beginning and end of both events. It clearly appeared that the whole image of the star was fainting during the dimming events, thus confirming that its origin is neither related to background objects occultation nor associated with any instrumental PSF motion.

\section{Models}

We can try to explain the repeated photometric event observed 928 days apart. The observed variations correspond to a dip in the star brightness by about $0.1 \%$, which lasted for about 4.4 days. In the context of a star that always shows multiple photometric variations in the form of brightness decrease, any other decrease in the star brightness suggests an explanation by the transit of a partially occulting body. With that in mind, the duration of the event ( $\sim 5$ days) is puzzling. With a possible period of 928 days, and assuming 1.4 solar mass for the F3V central star, the corresponding semi-major axis is 2.1 au and the orbital velocity on a circular orbit is $24.4 \mathrm{~km} \mathrm{~s}^{-1}$. At this transiting velocity, the maximum transit time in front of a $R_{*}=1.3 R_{\odot}$ star is about $10.3 \mathrm{~h}$. Even on a highly eccentric orbit and observed at apoastron, the transit of a body on a 928-day period orbit cannot last longer than $14.6 \mathrm{~h}$. Therefore, the photometric events of 4.4 days can be explained by the transit of an occulting body only if this body is significantly larger in size than the star; in this case, the duration of the transit is related to the size of the object itself.

The main scenario for explaining the other deeper dips in the KIC 8462852 light curve invokes the transit of trains of extrasolar comets (Boyajian et al. 2016; Bodman \& Quillen 2016) or planet fragments (Metzger et al. 2017). In fact, the photometric variations observed in KIC 8462852 light curve look like the spectroscopic variations observed in $\beta$ Pictoris, which can last several days and are interpreted by the transit of exocomets (Ferlet et al. 1987; Lagrange-Henri et al. 1992; Vidal-Madjar et al. 1994; Kiefer et al. 2014a). We explore this scenario in Sect. 5.1.

Nevertheless, keeping the idea of a transiting body, we can imagine another possible scenario to explain the repeated photometric events A and B. The straight ingress and egress slopes and the flat bottom of the light curve point towards the possibility that the transiting body can be a single body with a simple shape. Acknowledging that the Hill spheres of a massive planet can extend to several stellar radius in size, the transit of a ring system surrounding a giant planet could explain the observed photometric event $\mathrm{A}$ and $\mathrm{B}$, as in the light curve of 1SWASP J140747.93-394542.6 (also named J1407), an old star in the ScoCen OB association (Kenworthy \& Mamajek 2015); see also Lecavelier Des Etangs et al. (2017) and Aizawa et al. (2017) for other case studies of exoplanetary ring systems. This scenario is discussed in Sect. 5.2.

\subsection{Comets string model}

In the exocomets scenario, the duration of the transit event in the light curve implies that several comets passed in front of the star, within an extended string of several million kilometers long. While we do not aim to explore the whole range of possibilities to fit the events A and B light curves, we could use some of the exocomet tail transit signatures given, e.g. in the library of Lecavelier Des Etangs (1999), to show that a generic transit model of a few trailing exocomets can easily provide a satisfactory fit to the data.

As a reference light curve, we decided to use the light curve labelled “20_F_50_03_p4_00” in Lecavelier Des Etangs (1999) 
F. Kiefer et al.: A repeated transit signature in the light curve of KIC 8462852 with a possible 928-day period

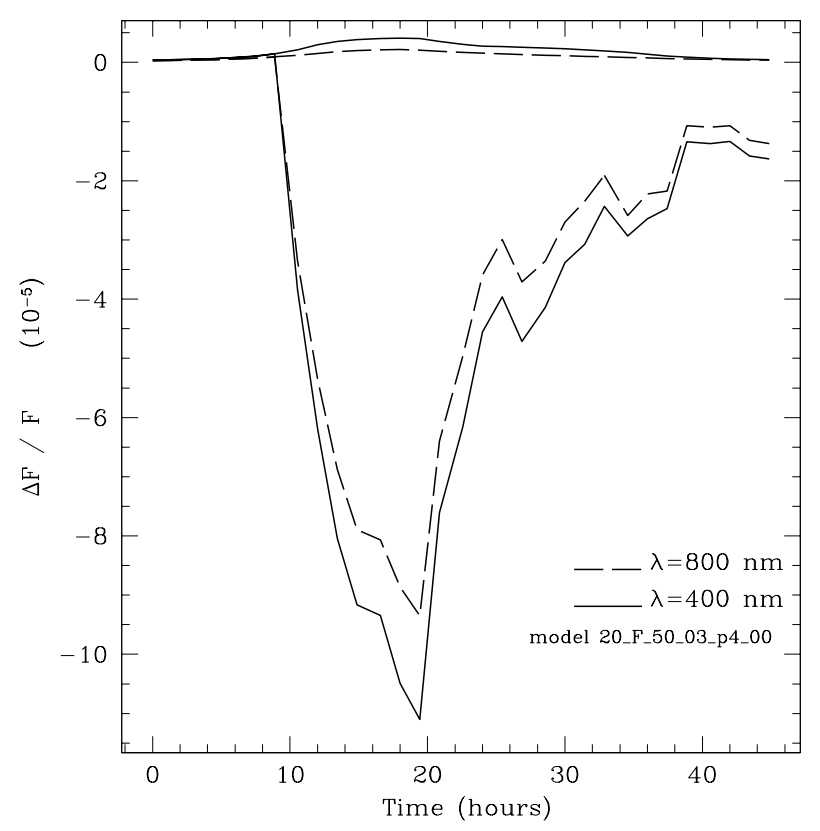

Fig. 9. Modelled exocomet transit light curve from Lecavelier Des Etangs (1999) for the case "20_F_50_03_p4_00", corresponding to an exocomet orbiting an $\mathrm{F}$ star with a periastron of $0.3 \mathrm{au}$, a longitude of periastron of $90^{\circ}$, and a production rate of $10^{5} \mathrm{~kg} \mathrm{~s}^{-1}$ at $1 \mathrm{au}$.

and plotted in Fig. 9. This plot is obtained through the simulation of cometary tails orbiting an F star with a periastron of $0.3 \mathrm{au}$, a longitude of periastron of $90^{\circ}$, and a production rate of $10^{5} \mathrm{~kg} \mathrm{~s}^{-1}$ at $1 \mathrm{au}$ (Lecavelier Des Etangs et al. 1999). It assumes a grain size distribution given by $\mathrm{d} n(s)=\left(1-s_{0} / s\right)^{m} s^{-n} \mathrm{~d} s$, with $s_{0}=$ $0.05 \mu \mathrm{m}, n=4.2, m=n\left(s_{\mathrm{p}}-s_{0}\right) / s_{0}$, and peaking at $s_{\mathrm{p}}=0.2 \mu \mathrm{m}$. This distribution is derived from observations in solar system comets at less than 0.5 au from the Sun. The physical model used to calculate the photometric transit signatures of exocomet tails is discussed in depth in Lecavelier Des Etangs et al. (1999).

The choice of the characteristics (the orbit and dust production rate) of the specific exocomet for the reference light curve is not critical because all the transit light curves show a similar triangular shape. At a fixed distance to the star, the transit depth of an individual light curve is constrained by the dust production rate, and the duration is mainly related to the longitude of the periastron. The depth of the global light curve resulting from the transit of a string of several exocomets therefore depends on the production rate of each exocomet. However, the duration of the global light curve is not constrained by the duration of each individual transit, but by the spread of the transit time of each exocomet.

To simplify the fit to the data, we approximated the reference light curve of a single comet by a piecewise linear function. Each individual exocomet light curve is defined by two parameters: the time of mid-transit, $T_{0}$, and the maximum occultation depth, $\Delta F / F$. Exploring the library of Lecavelier Des Etangs (1999), we find that in the range $10^{4}-10^{6} \mathrm{~kg} \mathrm{~s}^{-1}$ the maximum occultation depth is related to the dust production rate, $\dot{M}$, by $\log _{10} \dot{M} /\left(1 \mathrm{~kg} \mathrm{~s}^{-1}\right)=5+1.25 \times \log _{10}\left(\Delta F / F / 10^{-4}\right)$.

We fitted the average light curve of events $\mathrm{A}$ and $\mathrm{B}$ with a combination of several individual light curves defined by $T_{0 k}$ and $\dot{M}_{k}$ for each comet $k$ of the string. With N comets in the string, the total number of parameters reach $2 N+1$ with two parameters per comet and one for the baseline level (slightly larger than 1). Given the possibly large number of parameters,

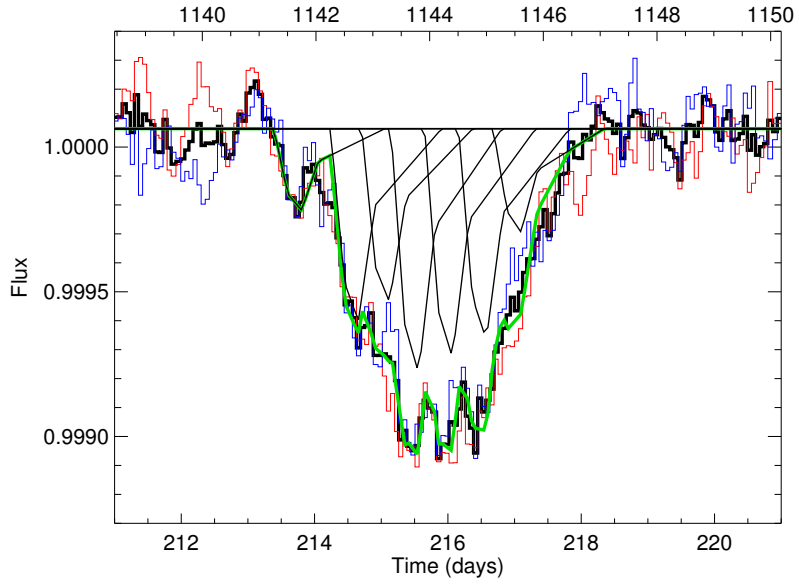

Fig. 10. Fit to the light curve using a string of 7 exocomets. The light curve of each exocomet is given by the thin black lines. The data of the events A and B are plotted with red and blue thin lines, and the coaddition of the two light curves is given by the thick black line. The best fit is plotted with the thick green line.

we used a Markov-chain Monte Carlo (MCMC) algorithm as a fitting procedure.

The best fit is obtained for seven comets, including the feature at the top of the signature left wing. It is plotted in Fig. 10 with the parameters given in Table 3 and plotted in Fig. 12. The dust production rates obtained for the comets are typical of Hale-Bopp type comets in the solar system, i.e. between $10^{5}$ and $10^{6} \mathrm{~kg} \mathrm{~s}^{-1}$ (Huang et al. 2000).

If we consider that we are actually overfitting stellar variations, we could accept a poorer fit with residuals of the order of the mean amplitude of the stellar variations. In this case, five comets are sufficient to fit the average light curve satisfyingly. An example of such a fit is shown in Fig. 11 with the parameters given in Table 3 and plotted in Fig. 12. Here we used a different longitude of periastron of $112.5^{\circ}$ and a different grain size distribution labelled "50" in Lecavelier Des Etangs (1999), peaking at $0.5 \mu \mathrm{m}$. This shows that the observations cannot constrain the properties of the comets and that the model of the comets can easily explain the data without any fine-tuning of parameters. Therefore, the values given in Table 3 should not be considered as measurements on existing bodies, but as possible values for a generic model of a string of exocomets.

Interestingly, both resulting models are reminiscent of the case of the solar system comet Shoemaker-Levy 9 (SL9). The bottom panel of Fig. 12 shows the distribution of diameters, $D$ (in log-space), with respect to timing of impact with Jupiter of all 21 fragments of SL9 (Hammel et al. 1995; Chodas \& Yeomans 1996; Crawford 1997). Since the dust production rate is proportional to the surface of the nucleus (all other things equal), $\log D$ of SL9 fragments could be compared to $\log \dot{M}$ of events A and B comets (Fig. 12, top-panel). We see that in both cases, the distribution of size (evaporation rate) is mainly flat with decreasing size of the comet nuclei at the head and tail of the fragments string. This tentatively suggests events A and B could be the break-up remnants of a bigger body along its orbit. If the periodicity of this transit is confirmed later, non-gravitational effects should be properly modelled to take into account a slow relative drift of the fragments. 


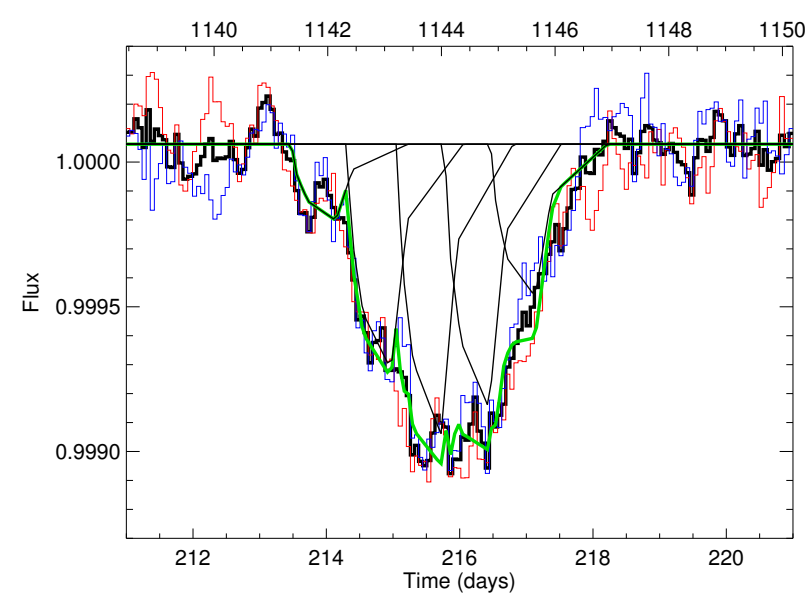

Fig. 11. Same as Fig. 10 using a string of 5 exocomets.

Table 3. Best-fit parameters for the models with the transit of 7 or 5 exocomets.

\begin{tabular}{lcc}
\hline \hline Comet & $\begin{array}{c}\text { Transit time } \\
T_{0} \\
\text { (day) }\end{array}$ & $\begin{array}{c}\text { Dust production rate } \\
\dot{M}\end{array}$ \\
& \multicolumn{2}{c}{7 comets model } \\
& \multicolumn{3}{c}{$5.56 \pm 0.18$} \\
1 & $213.39 \pm 0.11$ & $5.03 \pm 0.11$ \\
2 & $214.24 \pm 0.07$ & $5.97 \pm 0.12$ \\
3 & $214.71 \pm 0.11$ & $6.16 \pm 0.09$ \\
4 & $215.14 \pm 0.06$ & $6.12 \pm 0.08$ \\
5 & $215.65 \pm 0.05$ & $6.08 \pm 0.08$ \\
6 & $216.16 \pm 0.06$ & $5.69 \pm 0.16$ \\
7 & $216.68 \pm 0.12$ & $5.43 \pm 0.17$ \\
\hline \multicolumn{3}{c}{5 comets model } \\
1 & $213.48 \pm 0.13$ & $6.02 \pm 0.06$ \\
2 & $214.30 \pm 0.05$ & $6.15 \pm 0.05$ \\
3 & $215.05 \pm 0.05$ & $6.10 \pm 0.06$ \\
4 & $215.77 \pm 0.05$ & $5.80 \pm 0.10$ \\
5 & $216.46 \pm 0.08$ &
\end{tabular}

Notes. The error bars correspond to $3 \sigma$ and were estimated using MCMC. The central transit time, $T_{0}$, is given for event A; a constant of 928.25 days must be added for event B.

\subsection{Planetary ring model}

Here we discuss another possible scenario consisting in the transit of a giant ring system surrounding a planet with a 928-day orbital period (2.1 au semi-major axis). Indeed, a ring system can be stable within half a Hill-sphere radius of a planet. Around a massive planet the Hill sphere can extend up to several stellar radii in size; therefore rings (Kenworthy \& Mamajek 2015; Lecavelier Des Etangs et al. 2017; Aizawa et al. 2017) or dust envelopes, such as Fomalhaut b (Kalas et al. 2008), can be large enough that the transit duration can reach up to a few days. For instance, the Hill sphere of Jupiter extends up to 0.34 au $\left(73 R_{\odot}\right)$.

To model this scenario, we take the reference frame linked to the planet and consider that the star transits behind the rings. To simplify the problem, we assumed that the planet moves on a circular orbit at $2.1 \mathrm{au}\left(v_{\text {transit }}=24.4 \mathrm{~km} \mathrm{~s}^{-1}\right)$ and that the rings are seen face-on. We considered two simple models of rings and fit
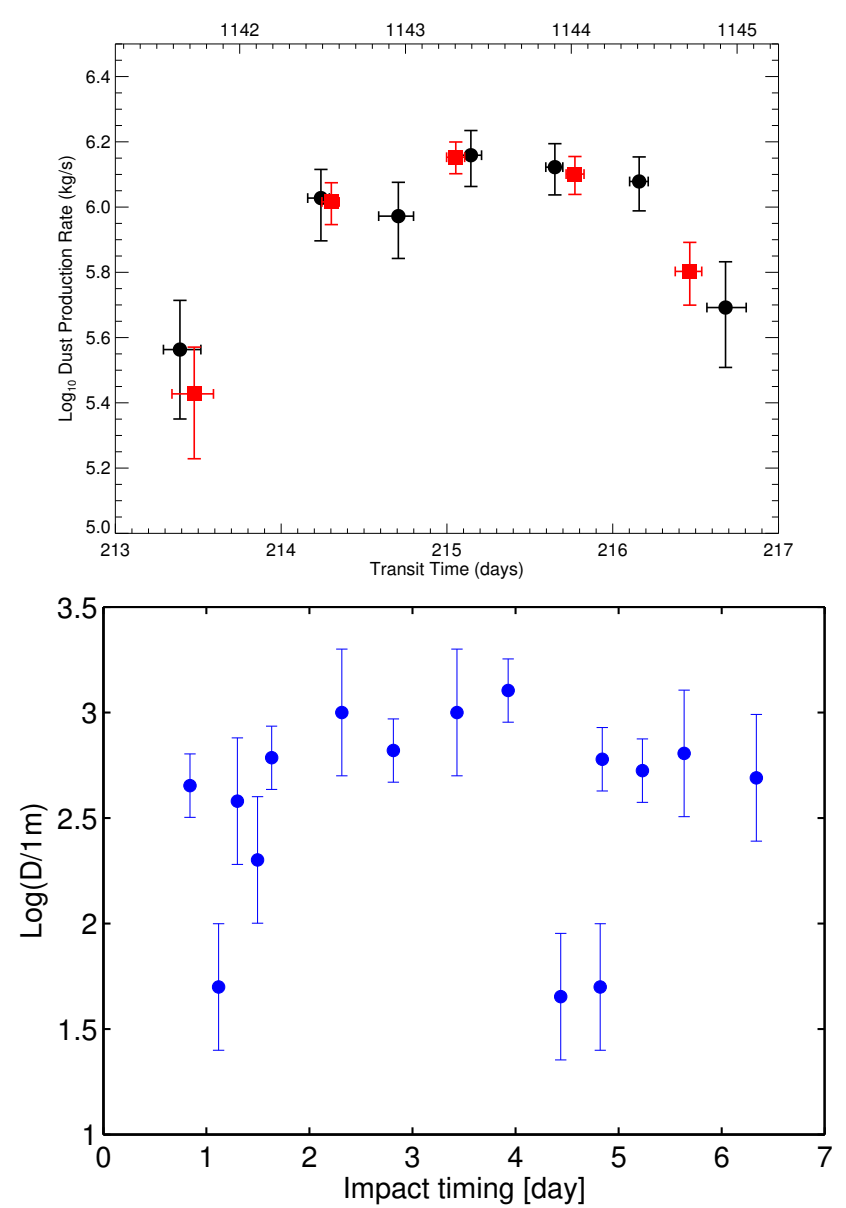

Fig. 12. Top panel: dust production rates and transit times of the transiting bodies for the 7 comet model (black dots; Fig. 10) and the 5 comet model (red squares; Fig. 11). Bottom panel: Shoemaker-Levy 9 fragments diameter vs. epochs of impact with the atmosphere of Jupiter.

these models to the data using Levenberg-Marquardt minimization of $\chi^{2}$ :

1. The first model consists in a large circular homogenous, constant opacity, ring with a non-zero impact parameter of the trajectory of the star behind the ring during the transit (Fig. 13, left panel). In this case, the signature of the transit is round. The data are best fitted with a ring exterior diameter of $8.8 R_{\star}$, an impact parameter of $8.5 R_{\star}$ and an extinction $\tau=0.0014$. Nonetheless, this model does not provide a good fit to the data, which show straight wings and a flat bottom.

2 . In the second model, the ring is made of an inner core of constant opacity for $r<R_{\text {const }}$ and an external ring with an extinction decreasing with the distance to the star following $\propto r^{-\alpha}$ for $r>R_{\text {const }}$ (Fig. 13, right panel). As can be seen in the figure, this model provides a much better fit to the data. Using a zero impact parameter, the best fit is found with an outer radius of $4.86 \pm 0.15 R_{\star}$, an interior core of radius $R_{\text {const }}=1.91 \pm 0.03 R_{\star}$ with constant extinction $\tau=(9.9 \pm$ $0.1) \times 10^{-4}$, and an extinction parameter $\alpha=1.70 \pm 0.06$.

We tried more sophisticated models by introducing elliptical rings, a non-zero impact parameter, and a non-zero position angle of the ellipse major-axis with respect to the transit direction (model \#3 in Table 4). The improvement of the fit is significant but only indicates that the rings as seen from Earth are likely elliptic $(e>0.8)$ and not aligned with the transit direction. This is in accordance with the observed asymmetry on the slopes of the left and right wings, as explained in Sect. 3. Since the projection 
F. Kiefer et al.: A repeated transit signature in the light curve of KIC 8462852 with a possible 928-day period
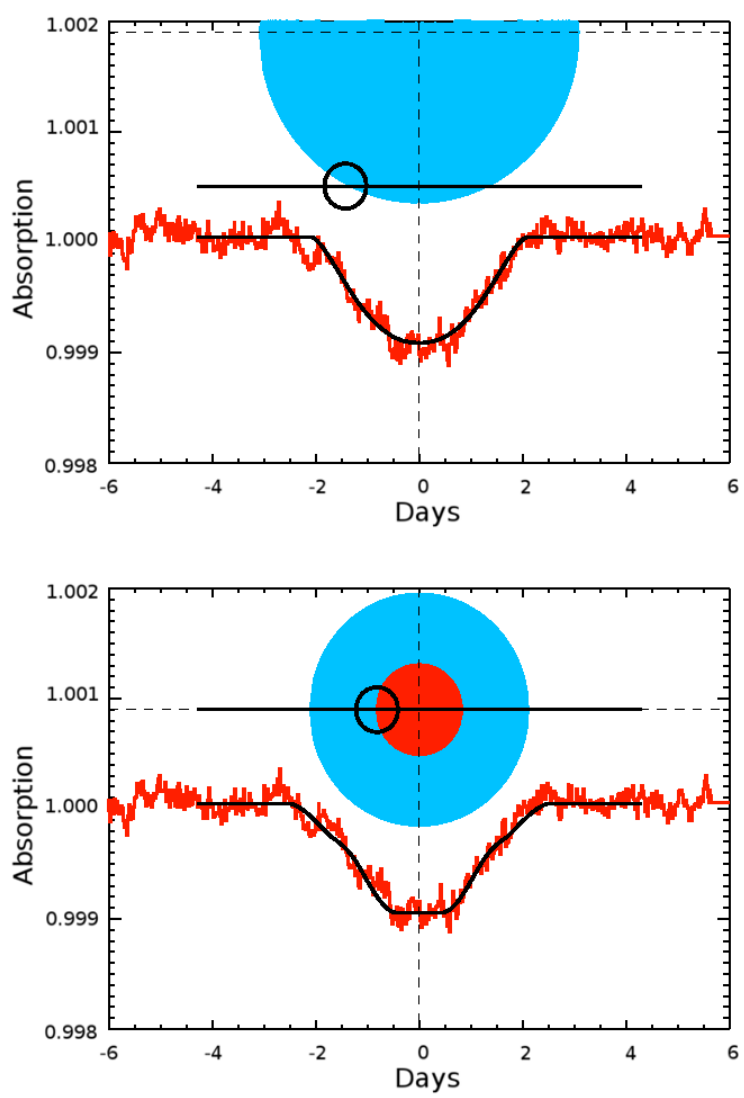

Fig. 13. Fit to the average light curve of events A and B (in red) by the two models of the occultation of KIC 8462852 by a planetary ring, as presented in Sect. 5 (black curves). Top panel: homogeneous circular ring with a constant opacity and a non-zero impact parameter. Bottom panel: circular ring with a constant opacity in the centre (red area) and a decreasing opacity with distance following a $r^{-\alpha}$ law in the external ring (blue area). The impact parameter is fixed to 0 . The black circle represents the edge of stellar disk.

of an inclined circle is an ellipse, the eccentric solution corresponds to a circular ring system inclined with respect to the plane of the sky at angle $\theta(=\arcsin e)>53^{\circ}$.

Interestingly, Ballesteros et al. (2018) recently proposed that the two main dips (D800 and D1500) of KIC 8462852 could be related to a ring planet on a 12-year orbit with trailing trojans at the L5 point. If true, KIC 8462852 might be the first exoplanetary system with two ring planets detected.

\section{Observing future events}

With the last event on BJD 2455977.15 and assuming periodicity with $P=t_{\mathrm{B}}-t_{\mathrm{A}}=928.25 \pm 0.25$ days, the phenomenon is expected to repeat itself every $t_{\mathrm{B}}+N \times P$. The occurrence timing closest to the present date is for $N=2$ (event D) with

$T_{\mathrm{D}}=2457833.65 \pm 0.80$

or between 20 March 2017 at 07:55 UT

and 21 March 2017 at 23:17 UT.

The beginning-of-ingress and end-of-egress timings were also estimated. Table 5 summarizes this information.

We planned to observe KIC 8462852 between 19 March and 23 March 2017 in photometry and/or spectroscopy. Unfortunately, HST and Spitzer were both unable to point at KIC 8462852 on these dates. Current ground-based photometry
Table 4. Table of $\chi^{2}$ and BIC of the different ring models proposed in the text.

\begin{tabular}{|c|c|c|c|c|}
\hline Models & $N_{\text {param }}$ & $N_{\text {DOF }}$ & $\chi^{2}$ & $\mathrm{BIC}$ \\
\hline $\begin{array}{l}\# 1 \text {. Circular ring } \\
\tau \text { constant }\end{array}$ & 5 & 430 & 590.0 & 620.4 \\
\hline $\begin{array}{l}\# 2 . \text { Circular rings } \\
r>R_{\text {const }}, \tau \propto r^{-\alpha} \\
r<R_{\text {const }}, \tau \text { constant }\end{array}$ & 6 & 429 & 550.1 & 586.6 \\
\hline $\begin{array}{l}\# 3 \text {. Elliptic rings } \\
r>R_{\text {const }}, \tau \propto r^{-\alpha} \\
r<R_{\text {const }}, \tau \text { constant } \\
e>0.8 \\
\text { Impact parameter } \neq 0 \\
\text { Position angle } \neq 0\end{array}$ & 9 & 426 & 515.6 & 570.3 \\
\hline
\end{tabular}

Notes. The value $\tau$ is the extinction.

Table 5. Timing and ephemeris of transit events with $P=928.25$ days starting from event B at $t_{\mathrm{B}}=1144$ days past Kepler initial epoch at MJD 2454833.

\begin{tabular}{lll}
\hline \hline \multicolumn{1}{c}{ MJD } & \multicolumn{1}{c}{ UT date } \\
\hline \multicolumn{2}{c}{ Most recent event in the past at $t_{\mathrm{B}}+2 \times P($ event D) } \\
& & \\
$T_{\text {ingress }}$ & $2457832.40 \pm 0.70$ & $19 / 03 / 17(04: 48)$ \\
& & $\rightarrow 20 / 03 / 17(14: 24)$ \\
$T_{\text {centroid }}$ & $2457833.65 \pm 0.80$ & $20 / 03 / 17(07: 55)$ \\
& & $\rightarrow 21 / 03 / 17(23: 17)$ \\
$T_{\text {egress }}$ & $2457835.70 \pm 0.60$ & $22 / 03 / 17(14: 24)$ \\
& & $\rightarrow 23 / 03 / 17(19: 12)$
\end{tabular}

Next event in the future at $t_{\mathrm{B}}+3 \times P($ event $\mathrm{E})$

$$
\begin{array}{lll}
T_{\text {ingress }} & 2458760.65 \pm 0.74 & 03 / 10 / 19(09: 30) \\
& & \rightarrow 04 / 10 / 19(21: 30) \\
T_{\text {centroid }} & 2458761.90 \pm 0.84 & 04 / 10 / 19(13: 00) \\
T_{\text {egress }} & 2458763.95 \pm 0.64 & \rightarrow 06 / 10 / 19 / 19(06: 00) \\
& & \rightarrow 08 / 10 / 19(02: 30)
\end{array}
$$

is not sensitive and stable enough to confirm a $0.1 \%$ deep transit signature lasting several days. We attempted ground-based spectroscopy since in case of exocomet transit, variable $\mathrm{Na}$ I or Ca II features could be expected in the KIC 8462852 spectrum (Kiefer et al. 2014a,b; Beust et al. 1990; Ferlet et al. 1987). We therefore planned observations of the star with the SOPHIE spectrograph installed on the $1.93 \mathrm{~m}$ telescope of Observatoire de Hautes-Provence (Perruchot et al. 2008; Bouchy et al. 2009) between 15 and 26 March 2017.

Unfortunately, bad weather conditions prevented us from observing KIC 8462852 after the 19 March 2017. We were able to collect good quality spectra of KIC 8462852 on 15, 16, 17, and 19 March between 03:30 and 03:45 UT. The median Na I spectrum of KIC 8462852 observed with SOPHIE between these four dates is plotted in Fig. 14. On the right-hand side of the stellar $\mathrm{Na}$ I doublet lines, we detected an emission feature, which is also observed in the simultaneous sky-background spectrum obtained through the second aperture of the spectrograph (fiber B). It is identified as geocoronal emission from atmosphere of the Earth. We subtracted this feature from all $\mathrm{Na}$ I spectra by fitting out the 


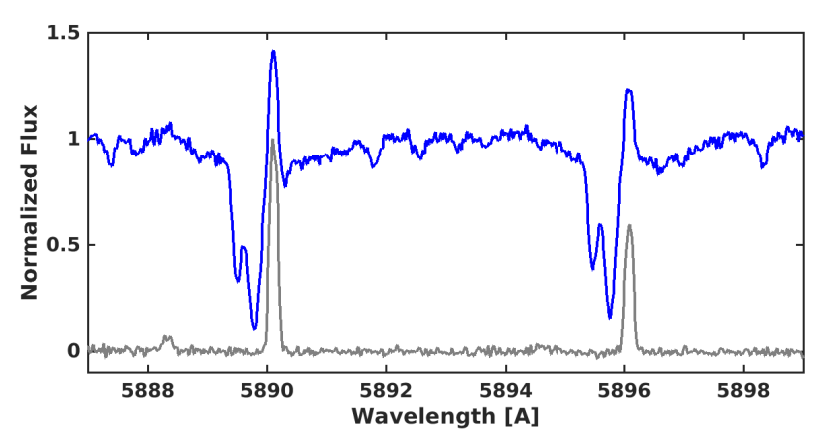

Fig. 14. Na I spectra of KIC 8462852. In blue, the average of the spectra collected through fiber A of the SOPHIE spectrograph, on the 15, 16, 17 and 19 March 2017. In grey, the average sky-background spectrum taken simultaneously with each star's spectrum on fiber B. The emission line seen on fiber A and B is clearly identified as geocoronal sodium emission. The double peak feature on the left of the telluric emission is most probably of interstellar absorption origin, since no counterpart is observed in the Ca II spectrum at the star radial velocity (see Fig. 16).

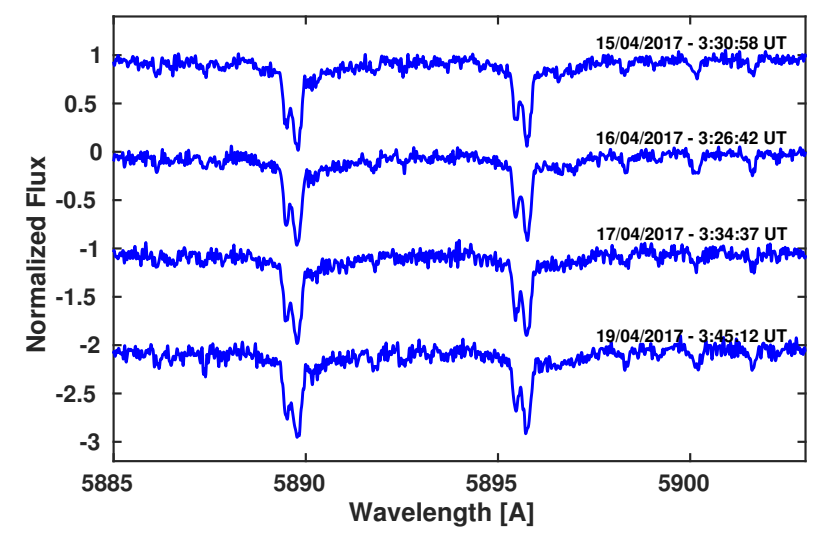

Fig. 15. Comparison of the Na I spectra of KIC 8462852 ordinated by increasing dates from top to bottom. The sky spectrum obtained simultaneously in fiber B has been fitted out of the original spectra (see Fig. 14).

sky spectrum. As can be seen in Fig. 15, the resulting Na I spectrum is totally quiet throughout the four days. It only presents a stable double peak absorption line, which is most likely of interstellar origin, since no counterpart is observed in the Ca II spectrum at the star radial velocity (Fig. 16). Similarly the Ca II doublet spectrum of KIC 8462852 does not present any variable features between 15-19 March.

Nevertheless, the predicted time of ingress is just after the observation dates. At the top of the signature left wing is 19 March at 03:45 (UT) before the predicted timing of ingress (19 March 04:48 UT). Therefore, the absence of observed features cannot exclude that significant absorption occurred in the KIC 8462852 spectrum during the transit. The observed spectra could neither confirm nor refute the periodicity of these transit events.

Assuming periodicity, the next event is predicted to occur between 3-8 October 2019 with ingress, centroid, and egress timings given in Table 5. New observations of KIC 8462852 between 3-8 October 2019 in both photometry and spectroscopy, with Spitzer, Cheops, HST, JWST, and ground-based spectroscopes, are strongly encouraged. They should allow confirmation or refutation of the periodicity in the observed photometric event.

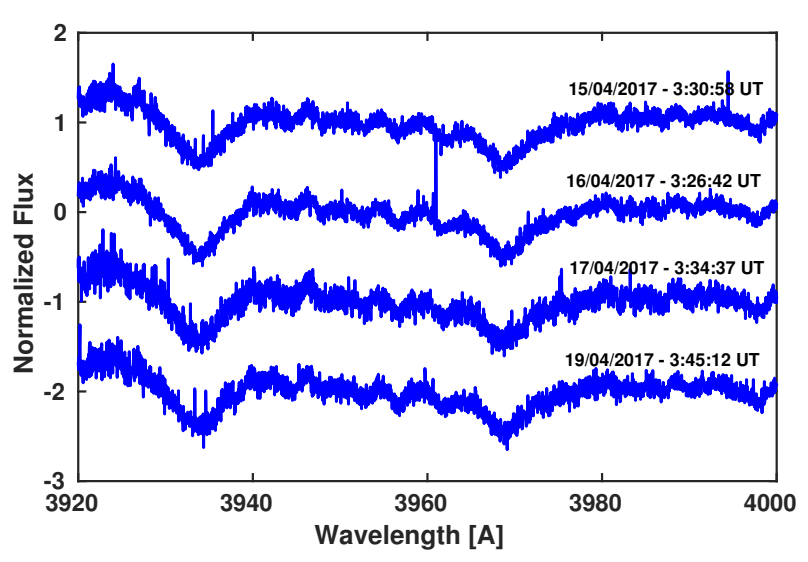

Fig. 16. Comparison of the Ca II spectra of KIC 8462852 ordinated by increasing dates from top to bottom, with 3-pixel binning. As can be seen, there are no spectral signatures of transient phenomenon in these spectra.

\section{Conclusions}

After a careful detrending of the Kepler light curve of the peculiar star KIC 8462852, we identified among 22 signatures, two strickingly similar shallow absorptions with a separation of 928.25 days (event $\mathrm{A}$ and $\mathrm{B}$ ). These two events presented $0.1 \%$ deep stellar flux variations with duration of 4.4 days, which is consistent with the transit of a single or a few objects with a 928-day orbital period.

We thoroughly verified the different possible sources of systematics that could have produced the transit-like signatures of event A and B. We conclude that these two events are certainly of astrophysical origin and occurred in the system of KIC 8462852.

We found that two scenarios could well reproduce the transit light curve of events A and B. They consist in the occultation of the star by two kinds of objects:

1. A string of a half dozen exocomets orbiting at a distance $\gtrsim 0.3 \mathrm{au}$ with evaporation rates similar to comet Hale-Bopp and scattered along their common orbit much like the 1994 Shoemaker-Levy 9 fragments.

2. An extended ring system surrounding a planet orbiting at 2.1 au from the star and composed of a constant opacity interior ring and an exterior ring with decreasing opacity towards larger radius.

It should be mentioned that the main argument against the exocomet scenario for KIC 8462852 dimming events is the absence of any detectable IR excess. This is an important problem that will always lead to risky comparison with other emblematic exocomet hosts such as $\beta$ Pic. These stars are all young $(<100 \mathrm{Myr})$ with strong Vega-like excess and thus massive debris disk. The age of KIC 8462852 ( $1 \mathrm{Gyr}$ ) would explain well the lack of IR excess, yet how the vaporization of the remaining small bodies would fit below the detection level remains to be explained. In fact, Boyajian et al. (2016) showed that dust clouds of the mass of a fully vaporized Hale-Bopp comet, as needed to explain the strongest dips of the KIC 8462852 light curve, are not expected to produce visible IR emission as long as the distance of the clouds is greater than $0.2 \mathrm{au}$. This happens to be the case in the exocomets string model proposed here.

This is the first strong evidence for a periodic signal coming from KIC 8462852. All the other dimmings present irregular behaviour with apparently uncorrelated timings. If periodic, our discovery opens a gate for the in-depth characterization of 
a collection of objects present around this star. Assuming periodicity, we predict that the next event to happen will occur between 3-8 October 2019. The observation of KIC 8462852 at these dates will confirm or deny the 928.25-day period, and hopefully will allow us to discriminate between the two scenarios proposed in this paper.

Acknowledgements. We thank the anonymous referee for a careful reading of our manuscript and insightful comments and suggestions. This work has been supported by the Centre National des Etudes Spatiales (CNES). We acknowledge the support of the French Agence Nationale de la Recherche (ANR), unde programme ANR-12-BS05-0012 "Exo-Atmos". V.B. acknowledges the financial support of the Swiss National Science Foundation. This paper includes data collected by the Kepler NASA mission and with the SOPHIE spectrograph on the $1.93 \mathrm{~m}$ telescope at Observatoire de Haute-Provence (CNRS). Funding for the Kepler mission is provided by the NASA Science Mission directorate.

\section{References}

Abeysekara, A. U., Archambault, S., Archer, A., et al. 2016, ApJ, 818, L33 Aizawa, M., Uehara, S., Masuda, K., Kawahara, H., \& Suto, Y. 2017, AJ, 153, 193

Ballesteros, F. J., Arnalte-Mur, P., Fernandez-Soto, A., \& Martinez, V. J. 2018, MNRAS, 473, L21

Beust, H., Vidal-Madjar, A., Ferlet, R., \& Lagrange-Henri, A. M. 1990, A\&A, 236, 202

Bodman, E. H. L., \& Quillen, A. 2016, ApJ, 819, L34

Borucki, W. J., Koch, D., Basri, G., et al. 2010, Science, 327, 977

Bouchy, F., Hébrard, G., Udry, S., et al. 2009, A\&A, 505, 853

Boyajian, T. S., LaCourse, D. M., Rappaport, S. A., et al. 2016, MNRAS, 457, 3988

Chodas, P. W., \& Yeomans, D. K. 1996, The Collision of Comet

Shoemaker-Levy 9 and Jupiter, IAU Colloq., 156, 1

Crawford, D. A. 1997, Ann. N. Y. Acad. Sci., 822, 155

Ferlet, R., Vidal-Madjar, A., \& Hobbs, L. M. 1987, A\&A, 185, 267

Hammel, H. B., Beebe, R. F., Ingersoll, A. P., et al. 1995, Science, 267, 1288
Harp, G. R., Richards, J., Shostak, S., et al. 2016, ApJ, 825, 155

Hippke, M., \& Angerhausen, D. 2016, ArXiv e-prints [arXiv: 1609. 05492]

Hippke, M., Angerhausen, D., Lund, M. B., Pepper, J., \& Stassun, K. G. 2016, ApJ, 825, 73

Hippke, M., Kroll, P., Matthai, F., et al. 2017, ApJ, 837, 85

Huang, K., Hu, J., \& Zhou, H. 2000, Proc. Observations and Physical Studies of Comet Hale-Bopp and Other Comets, eds. Z. Junliang \& W. Ningshan (Shanghai: Ke Xue Ji Sju Chu Ban She), 44

Kalas, P., Graham, J. R., Chiang, E., et al. 2008, Science, 322, 1345

Kenworthy, M. A., \& Mamajek, E. E. 2015, ApJ, 800, 126

Kiefer, F., Lecavelier des Etangs, A., Boissier, J., et al. 2014a, Nature, 514, 462

Kiefer, F., Lecavelier des Etangs, A., Augereau, J.-C., et al. 2014b, A\&A, 561, L10

Kinemuchi, K., Barclay, T., Fanelli, M., et al. 2012, PASP, 124, 963

Lagrange-Henri, A. M., Beust, H., Ferlet, R., Vidal-Madjar, A., \& Hobbs, L. M. 1990, A\&A, 227, L13

Lagrange-Henri, A. M., Gosset, E., Beust, H., Ferlet, R., \& Vidal-Madjar, A. 1992, A\&A, 264, 637

Lecavelier Des Etangs, A. 1999, A\&AS, 140, 15

Lecavelier Des Etangs, A., Vidal-Madjar, A., \& Ferlet, R. 1999, A\&A, 343, 916 Lecavelier Des Etangs, A., Hébrard, G., Blandin, S., et al. 2017, A\&A, 603, A115

Lisse, C. M., Sitko, M. L., \& Marengo, M. 2015, ApJ, 815, L27

Lund, M. B., Pepper, J., Stassun, K. G., Hippke, M., \& Angerhausen, D. 2016, ApJ, submitted [arXiv: 1605.02760 ]

Makarov, V. V., \& Goldin, A. 2016, ApJ, 833, 78

Marengo, M., Hulsebus, A., \& Willis, S. 2015, ApJ, 814, L15

Metzger, B. D., Shen, K. J., \& Stone, N. C. 2017, MNRAS, 468, 4399

Miles, B. E., Roberge, A., \& Welsh, B. 2016, ApJ, 824, 126

Montet, B. T., \& Simon, J. D. 2016, ApJ, 830, L39

Montgomery, S. L., \& Welsh, B. Y. 2012, PASP, 124, 1042

Neslušan, L., \& Budaj, J. 2017, A\&A, 600, A86

Perruchot, S., Kohler, D., Bouchy, F., et al. 2008, Proc. SPIE, 7014, 70140J Schaefer, B. E. 2016, ApJ, 822, L34

Schuetz, M., Vakoch, D. A., Shostak, S., \& Richards, J. 2016, ApJ, 825, L5 Smith, J. C., Stumpe, M. C., Van Cleve, J. E., et al. 2012, PASP, 124, 1000 Thompson, M. A., Scicluna, P., Kemper, F., et al. 2016, MNRAS, 458, L39 van Leeuwen, F., Evans, D. W., De Angeli, F., et al. 2017, A\&A, 599, A32

Vidal-Madjar, A., Lagrange-Henri, A.-M., Feldman, P. D., et al. 1994, A\&A, 290,245

Wright, J. T., \& Sigurdsson, S. 2016, ApJ, 829, L3 